\title{
Harnessing desktop computers for ab initio calculation of vibrational IR/Raman spectra of large molecules
}

\author{
SUBODH S KHIRE ${ }^{\mathrm{a}}$, NITYANANDA SAHU ${ }^{\mathrm{b}}$ and SHRIDHAR R GADRE ${ }^{\mathrm{a}, *}$ \\ anterdisciplinary School of Scientific Computing, Savitribai Phule Pune University, Pune 411007, India \\ ${ }^{\mathrm{b}}$ Max Planck Institute für Kohlenforschung, 45470 Mülheim an der Ruhr, Germany \\ E-mail: gadre@unipune.ac.in
}

MS received 11 September 2018; revised 10 October 2018; accepted 19 October 2018; published online 2 November 2018

\begin{abstract}
The requirement of huge computational resources makes quantum chemical investigations on large molecules prohibitively difficult. In particular, calculating the vibrational IR/Raman spectra of large molecules employing correlated $a b$ initio theory is a herculean task. The present article brings out the utility of our molecular tailoring approach (MTA)-based software for accurate yet economic spectral calculations employing one or more desktop computers. Hartree-Fock and density functional theory-based benchmark calculations on test cases containing over 175 atoms and over 2300 basis functions show excellent agreement with their full calculations (FC) counterparts with large savings in the computer time and memory/hard disk requirements. These savings are even more impressive at MP2 level of theory. Our MTA-based software thus represents an art-of-the-possible for computing vibrational IR/Raman spectra using a handful of desktop machines.
\end{abstract}

Keywords. Vibrational infrared (IR) and Raman spectra; Hartree-Fock (HF); Density Functional Theory (DFT); Møller-Plesset second-order perturbation theory (MP2); Molecular Tailoring Approach (MTA); large molecules.

\section{Introduction}

Advents in spectroscopy have made measurements of structures and properties of molecules and materials routinely possible. ${ }^{1}$ In particular, Infrared (IR) and Raman spectroscopy are commonly used techniques for exploring structures of large molecular systems. ${ }^{2,3}$ These explorations need to be supplemented or complemented by theoretical studies, e.g. quantum chemistry (QC)-, Monte Carlo (MC)-, molecular dynamics (MD)-based computations/simulations, etc. Among all of these, QC studies on molecular systems are quite reliable for a meaningful comparison with the corresponding experimental spectra. ${ }^{4}$

Within QC framework, several levels of theory are available, such as Hartree-Fock (HF), Density Functional Theory (DFT), Møller-Plesset second-order perturbation theory (MP2), etc., differing in the construction of wave function/electron density for solving the Schrödinger equation. These theories have to be executed in conjunction with a careful choice of basis set.
The computational cost (in terms of execution time) of a QC method is determined by the level of theory and number of basis functions, $N$. The hardware capability (such as memory, disk space, etc.) is also another limiting parameter for carrying out the calculation of energies and spectra of large molecules. The HF and DFT energy computations typically scale $O\left(N^{3}\right.$ or $\left.N^{4}\right)$ whereas correlated theories, e.g. MP2 and coupled cluster singles and doubles with perturbative triples viz. $\operatorname{CCSD}(\mathrm{T})$, scale as $O\left(N^{5}\right)$ and $O\left(N^{7}\right)$ respectively. ${ }^{5,6}$ The spectral calculations are even more demanding in terms of CPU time, memory and/or disk space. Pursuing such QC investigations requires huge computational resources and effective parallel algorithms. ${ }^{7}$ The required hardware resources are not readily available at several academic/research institutions for everyday use.

To overcome the computational power requirements and to cut down the scaling complexities of the QC methods, several alternate routes have been proposed by researchers. Density-fitting (DF), Resolution of Identity (RI), Local and fragment-based methods are some of the

\footnotetext{
*For correspondence

Electronic supplementary material: The online version of this article (https://doi.org/10.1007/s12039-018-1568-3) contains supplementary material, which is available to authorized users.
} 
examples. ${ }^{8-11}$ However, the accuracy and efficiency of DF, RI and local methods need to be carefully benchmarked, especially for the calculation of the spectral properties. On account of simplicity in execution, ease in parallelization and high accuracy, ${ }^{12,13}$ fragment-based methods have gained popularity over the last decade. Nevertheless, it should be borne in mind that all such routes are approximations to canonical full calculations (FC) which demand huge computational resources.

Many fragmentation-based methods have been developed $^{12,14,15}$ in the quantum chemical literature since the 1970s. The central theme of all fragmentation-based methods is to divide the parent system into subsystems such that the latter can be readily treated computationally. Within the framework of such methods, the most crucial part is the a priori generation of quality fragments, assuring adequate accuracy and efficiency. Most of the fragmentation-based methods do not adequately address this inherent problem. Furthermore, most of these methods have been benchmarked only for energy calculation and geometry optimization. On account of extensive computations involved therein (viz., the Hessian matrix, derivatives of dipole moment and polarizability tensor, etc.), the accurate theoretical treatment of vibrational IR and Raman spectra for large molecular systems is an arduous task even on large hardware. Moreover, such computations employing contemporary desktop/server computers with multi-core architecture (typically 8 cores, 64/128 GB RAM and 1 to 2 TB hard disk) are either impossible or extremely timeconsuming. In the present work, we demonstrate our home-built molecular tailoring approach (MTA)-based software showcasing the calculations of energies and vibrational (within harmonic approximation) IR/Raman spectra of large molecules employing a multi-core desktop machine. Details of MTA have been given in the earlier publications from our group, ${ }^{12,13}$ but a brief summary of MTA is given for the uninitiated reader in the following section.

\section{Computational methodology}

An indigenous fragmentation-based method, viz., MTA was developed in our group and employed for exploring energetics and spectral features of large molecules/molecular clusters at $a b$ initio level such as HF, DFT, MP2, etc. MTA is essentially based on the set inclusion-exclusion principle (SIEP). The spatially extended parent molecule under investigation is divided into a series of main and overlapping subsystems, named fragments. These fragments are subjected to the computation instead of the entire parent molecule. A desired electronic property, $\mathrm{P}$, of a parent molecule is then estimated by combining the properties of the individual fragments employing SIEP as an approximation, vide Eq. (1):

$$
\begin{aligned}
P= & \sum_{i} P^{F_{i}}-\sum_{i<j} P^{F_{i} \cap F_{j}}+\cdots \\
& +(-1)^{k} \sum_{i<j<\cdots<n} P^{F_{i} \cap F_{i} \cap \cdots \cap F_{n}}
\end{aligned}
$$

In Eq. (1), $\mathrm{P}^{\mathrm{Fi}}$ denotes the property associated with $\mathrm{i}^{\text {th }}$ fragment, $\mathrm{P}^{\mathrm{Fi} \cap \mathrm{Fj}}$ represents the value of property $\mathrm{P}$ of the binary overlap between the fragments $i$ and $j$ where as $k$ is the order of overlap between the fragments. On account of dividing the parent molecule into small fragments, computational expenses are steeply reduced. However, the estimate of the property P produced by the use of MTA carries some errors, essentially due to interatomic interactions that are completely missed out. Sharing the spirit of methods ${ }^{16,17}$ such as ONIOM, G2, etc., a grafting correction ${ }^{18}$ was introduced in 2012 with an aim to reduce the error occurring due to the missing interactions within MTA. In this correction, the contribution of the missing interatomic interactions due to fragmentation is estimated from the difference between the energies of MTA and FC calculations at a suitable lower basis set (LB), by keeping the fragmentation scheme unaltered.

$$
P^{H B}=P_{M T A}^{H B}+\left(P_{F C}^{L B}-P_{M T A}^{L B}\right)
$$

Here, $P^{H B}$ is the electronic property of the parent molecule estimated at higher basis set (HB) after effecting the grafting correction, $P_{M T A}^{H B}$ is the property computed by the MTA (using Eq. 1) procedure at the HB. $P_{F C}^{L B}$ and $P_{M T A}^{L B}$ are the electronic properties computed at the LB set by doing the Full and MTA calculation respectively.

The technical details of the software will be made available in a forthcoming publication. However, we give below brief highlights of the present algorithm. Following is the input needed for running the software, in an interactive mode. A sample input file is given in the Supplementary Information (TS-2).

1. The Cartesian coordinates (in $\AA$ ) of the parent molecule/ cluster (closed shell) in xyz format.

2. The Cartesian coordinates of the fragments.

3. Level of theory such as HF/DFT/MP2.

4. Lower basis set (LB), viz., the basis used for grafting.

5. Higher basis set (HB), viz., the basis at which the property estimation is desired.

All the keywords are to the given as per the format of the Gaussian program. On receiving the above inputs, our MTAbased software initiates the Gaussian program to extract the FC Hessian matrix and/or dipole- and polarizabilityderivative tensors at LB followed by MTA calculations [by SIEP of Eq. (1)] at LB and HB. With the use of Eq. (2), the difference between the FC- and MTA-Hessian matrix elements at LB is grafted to those generated by MTA at HB. The flowchart illustrating this algorithm is depicted in Figure 1. The Hessian matrix thus obtained is converted into mass weighted Hessian matrix for generating vibrational frequencies and the corresponding normal modes. In a similar manner, the dipole- and 


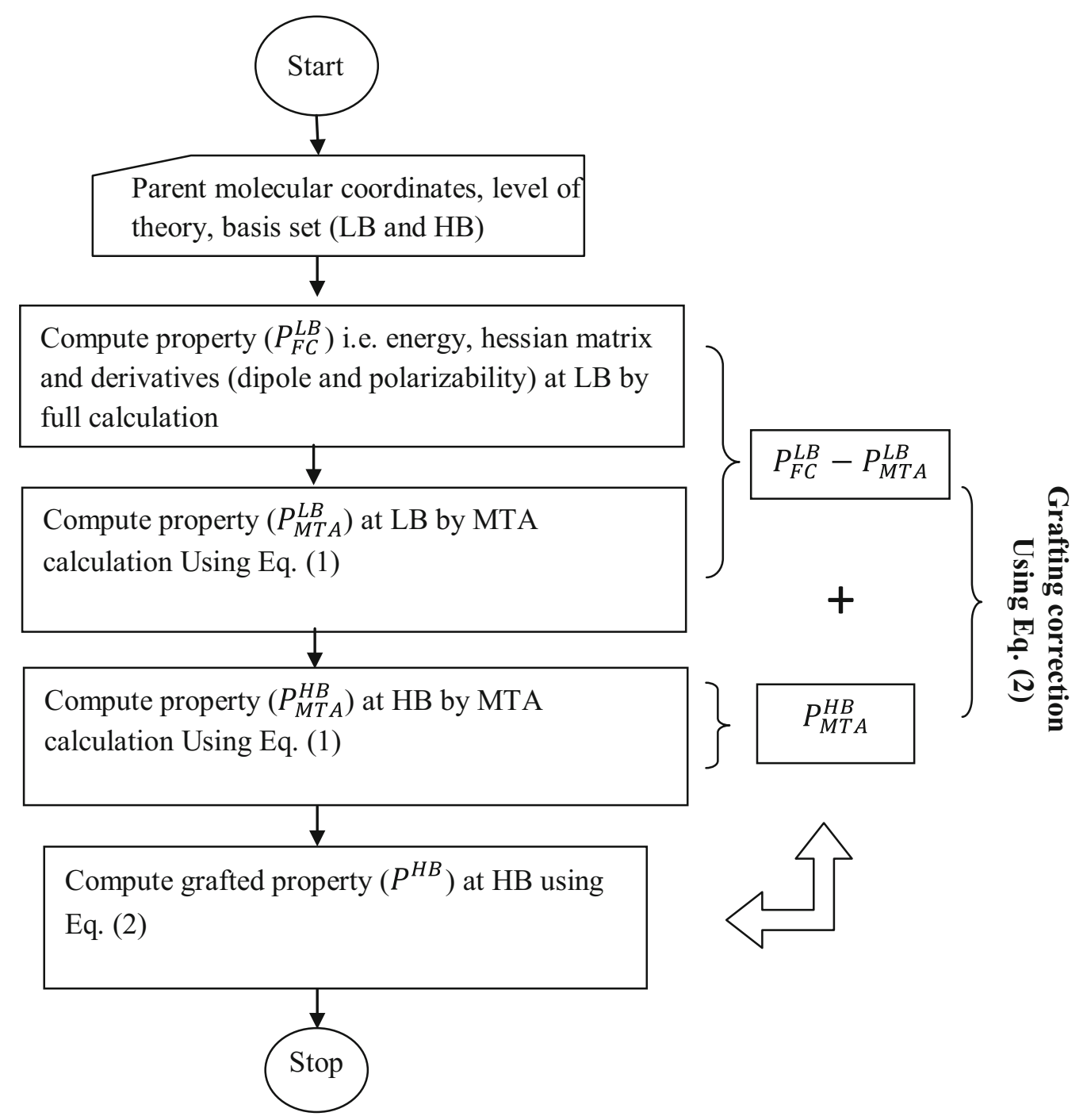

Figure 1. Flowchart of the automated MTA algorithm for calculating vibrational IR/Raman spectra of large molecular systems. See text for details.

polarizability-derivative tensors are generated and used for calculating IR/Raman intensities respectively. ${ }^{12,13}$

For all the test cases, fragmentation is done manually by the use of visualization software MeTAStudio. ${ }^{19}$ However, for the uninitiated user, the utility for automatic generation of fragmentation scheme is also available. ${ }^{20}$ The computations on fragments are carried out by employing Gaussian 16 software package. ${ }^{21}$ As stated in the previous section, several MTA-based calculations here are done on desktop computers with 4/8 cores and 8/64/128 GB RAM and 1 to 2 TB hard disk. For some larger systems, a 16 core computer with 128/256 GB RAM and upto 4 TB hard disk is used if required.

\section{Results and Discussion}

Table 1 displays the test cases along with a number of atoms, level of theory and basis sets employed
Table 1. Test systems (molecules/clusters), the corresponding level of theory (LT) and basis set, Number of atoms (NA) and Number of basis functions (NBF). See text for details.

\begin{tabular}{|c|c|c|c|}
\hline System & LT/Basis Set & NA & NBF \\
\hline$\left(\mathrm{CO}_{2}\right)_{25}$ & HF/aug-cc-pVDZ & 75 & 1725 \\
\hline$\alpha$-Cyclodextrin & B3LYP/6-31++G** & 126 & 1614 \\
\hline subst-acetamide & B3LYP/6-311++G** & 66 & 1025 \\
\hline Vancomycin & B3LYP/6-31++G** & 176 & 2377 \\
\hline $\mathrm{Fc} @ \mathrm{CB}[7]^{\mathrm{a}}$ & $\omega \mathrm{B} 97 \mathrm{X} / 6-31+\mathrm{G}^{*}$ & 147 & 1939 \\
\hline $\mathrm{Bz}-\left(\mathrm{H}_{2} \mathrm{O}\right)_{8}^{\mathrm{b}}$ & MP2/aug-cc-pVDZ & 36 & 520 \\
\hline$\beta$-carotene & MP2/aug-cc-pVDZ & 96 & 1424 \\
\hline
\end{tabular}

${ }^{\mathrm{a}}$ Geometry is adapted from Ref. ${ }^{28}$.

${ }^{\mathrm{b}}$ Geometry is taken from Ref. ${ }^{27}$.

for the calculations. Vibrational IR/Raman spectra are benchmarked at HF/DFT (with B3LYP and $\omega$ B97X functional) as well as at MP2 level of theory. Table 1 
Table 2. Test systems (molecules/clusters), level of theory (LT) and basis set, FC and MTA-based energies, viz. $\mathrm{E}_{\mathrm{FC}}$ and $\mathrm{E}_{\mathrm{MTA}}$ in a.u. whereas $\triangle \mathrm{E}$ is the absolute difference between $\mathrm{E}_{\mathrm{FC}}$ and $\mathrm{E}_{\mathrm{MTA}}$ energies in $\mathrm{mH}$. See text for details.

\begin{tabular}{lllcc}
\hline System & LT/Basis Set & \multicolumn{1}{c}{$\mathbf{E}_{\mathbf{F C}}$} & $\mathbf{E}_{\mathbf{M T A}}$ & $\mathbf{\Delta E}$ \\
\hline$\left(\mathrm{CO}_{2}\right)_{25}$ & HF/aug-cc-pVDZ & -4691.68084 & -4691.68024 & 0.60 \\
$\alpha$-Cyclodextrin & B3LYP/6-31++G** & -3664.79414 & -3664.79548 & 1.34 \\
Subst-acetamide & B3LYP/6-311++G** & -2023.47451 & -2023.47489 & 0.38 \\
Vancomycin & B3LYP/6-31++G** & -5779.06178 & -5779.06149 & 0.29 \\
Fc@CB[7] & wB97X/6-31+G* & -5862.22884 & -5862.22590 & 2.94 \\
Bz- $\left(\mathrm{H}_{2} \mathrm{O}\right)_{8}$ & MP2/aug-cc-pVDZ & -841.76510 & -841.76469 & 0.41 \\
$\beta$-Carotene & MP2/aug-cc-pVDZ & - & -1553.10494 & - \\
\hline
\end{tabular}

reveals that the test systems in the present work include closed-shell molecules/molecular clusters containing up to 180 (mostly) first-row atoms and/or 2400 basis functions at DFT levels. The test cases for MP2 level contain typically up to 96 first-row atoms and/or 1400 basis functions. In order to assess the quality of energies and the associated vibrational IR/Raman spectra generated by our MTA-based software, the results for few test cases are compared with their FC counterparts. For the molecular test cases, viz., $\alpha$-cyclodextrin, substituted acetamide, vancomycin and $\beta$-carotene, the initial geometries were taken from Refs. ${ }^{22-25}$ respectively. These geometries were further optimized respectively at B3LYP/6-31++G**, B3LYP/6-311++G**, B3LYP/6$31+\mathrm{G}^{*}$ and MP2/cc-pVDZ levels of theory. In the case of molecular clusters, the most stable $\left(\mathrm{CO}_{2}\right)_{25}$ structure generated through Monte Carlo procedure by Takeuchi ${ }^{26}$ and benzene-water cluster $\left(\mathrm{BZ}-\left(\mathrm{H}_{2} \mathrm{O}\right)_{8}\right)$ optimized at MP2/aug-cc-pVDZ as reported by Miliordos et al., ${ }^{27}$ are also selected as test cases. These geometries are taken up further for IR computations at HF and MP2 respectively levels of theory employing aug-cc-pVDZ (aVDZ) as HB. Furthermore, the test systems also include a host-guest complex of a ferrocenyl derivative with cucurbit[7]uril macrocycle (Fc@CB[7]). For this purpose, the initial geometry is taken from Ref. ${ }^{28}$ which is further optimized at $\omega \mathrm{B} 97 \mathrm{X} / 6-31++\mathrm{G}^{* *}$ level of theory.

Single point energy and spectral calculations are done at the level of theory and basis set specified in Table 1 . Before embarking on the MTA computations of vibrational IR/Raman spectra, it is felt worthwhile to assess the accuracy of the MTA energies vis-à-vis the respective FC values (whenever feasible). This comparison is shown in Table 2. It may be noticed from Table 2 that the grafted MTA energies obtained with the choice of appropriate LB, match their FC counterparts generally very well, with a typical error of 1 millihartree or less. This observation is in line with our recent study ${ }^{20}$ bringing out a sub-millihartree error in energies

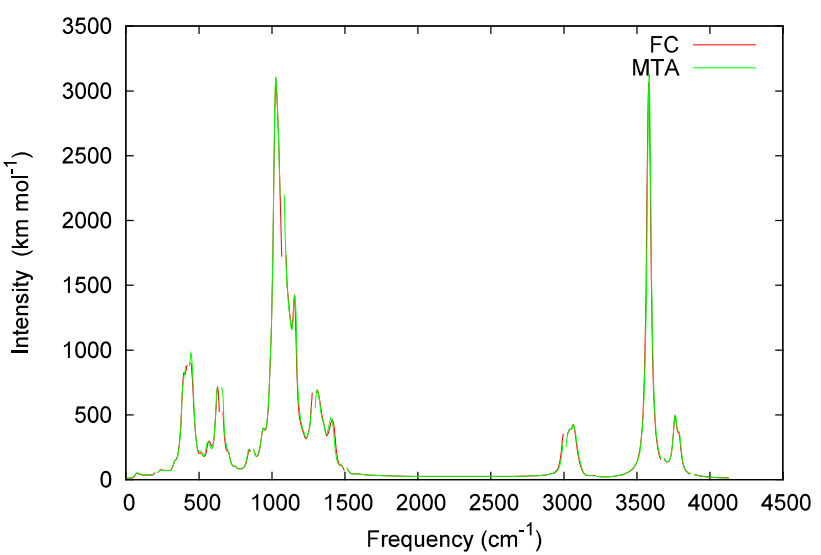

Figure 2. Superimposed MTA and FC vibrational IR spectra for $\alpha$-cyclodextrin calculated at B3LYP/6-31++G** level of theory. See text for details.

of large molecules or clusters. It may, however, be noted that MTA calculations represent a delicate balance of accuracy and efficacy. ${ }^{20}$ Thus, due to efficiency-based choosing of fragmentation scheme and use of LB (to ensure the possibility of doing FC at LB), a somewhat larger error is observed in the case of $\alpha$-cyclodextrin and Fc@CB[7].

With the desired accuracy of MTA energy data achieved, the calculations are taken further for computing the vibrational IR and Raman spectra of these molecules. It may be noted that without the fragmentation approach, such computations are extremely cumbersome especially using low-end hardware. For benchmarking purpose, vibrational IR spectra for some of the test cases, viz., $\alpha$-cyclodextrin, $\left(\mathrm{CO}_{2}\right)_{25}, \mathrm{Bz}-$ $\left(\mathrm{H}_{2} \mathrm{O}\right)_{8}$ and subst-acetamide at B3LYP, HF, MP2 and B3LYP level of theories respectively, are compared with the corresponding FC ones. Further, vibrational Raman spectrum for one of the test cases, viz., substacetamide has been compared with the corresponding FC Raman spectrum. For effecting the grafting procedure, cc-pVDZ (VDZ) is employed as LB when aVDZ is used as HB. For rest of the cases, 6-31G is used as LB. 


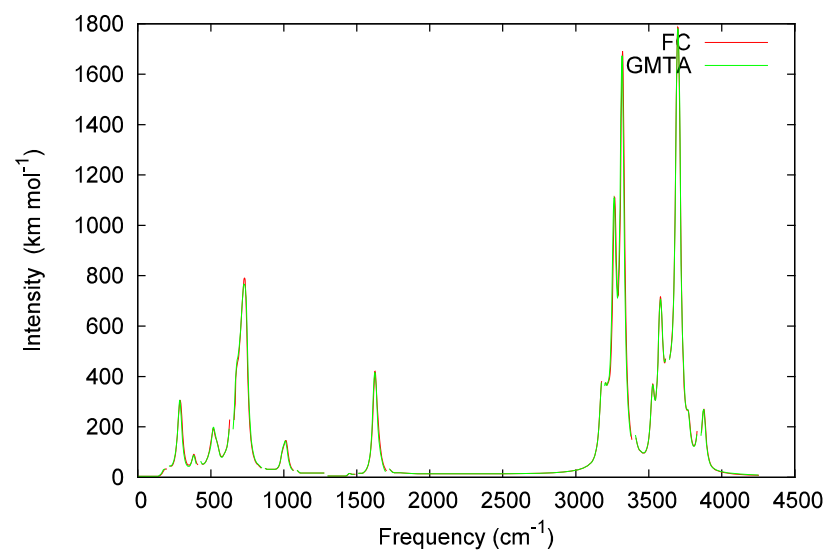

Figure 3. Superimposed MTA and FC vibrational IR spectra for $\mathrm{Bz}-\left(\mathrm{H}_{2} \mathrm{O}\right)_{8}$ calculated at MP2/aVDZ level of theory. See text for details.

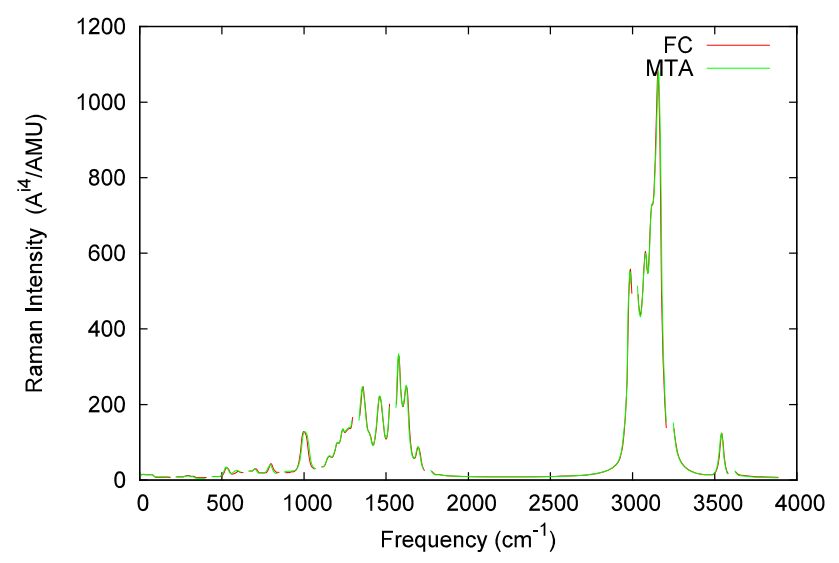

Figure 4. Comparison of MTA and FC vibrational IR spectra for subst-acetamide at B3LYP/6-311++G** level theory. See text for details.

Figure 2 shows the MTA and FC vibrational IR spectra broadened by the Gaussian with FWHM value of $20 \mathrm{~cm}^{-1}$ for $\alpha$-cyclodextrin calculated at B3LYP/6$31++\mathrm{G}^{* *}$ level theory. Figure 3 depicts MTA- along with FC-vibrational IR spectrum (appropriately broadened) for Bz- $\left(\mathrm{H}_{2} \mathrm{O}\right)_{8}$ complex at MP2/aVDZ theory. Similarly, Figure 4 shows a comparison of MTA and FC vibrational Raman spectrum for subst-acetamide. Within MTA, all the intense peaks are seen to be well preserved in comparison to the corresponding FC ones, with typical error in vibrational frequencies being less than $3 \mathrm{~cm}^{-1}$. In fact, a visual comparison reveals that the MTA- and FC spectra for the test cases ( $c f$. Figures $2,3,4)$ are almost indistinguishable from each other.

With this success in benchmarking, we generated the MTA spectra for rest of the test cases and are made available in the Supplementary Information. Table 3 reports the comparison of wall-clock times (total elapsed time calculated by taking the difference between job end and job start time) along with hardware employed, for MTA vis-à-vis FC spectral computations, in order to assess the efficacy of the MTA-based calculations. The FC spectral calculations of some test cases are not feasible on the indicated hardware, and the respective entries are marked with a '-'. Time gain is seen to be very substantial for all the test cases. Moreover, in the case of the test case of $\beta$-carotene, the vibrational IR spectrum at MP2/aVDZ is feasible only with the use of MTA. It is significant to note that in the case of Bz- $\left(\mathrm{H}_{2} \mathrm{O}\right)_{8}$, not only the MTA wall-clock time is smaller, but also the computational hardware used (8 core) is substantially lower (16 core for FC).

\section{Conclusions}

With the increasing sophistication in instrumental facilities, studies on vibrational IR and Raman spectra of molecules have become routinely possible. However,

Table 3. Test systems (molecules/clusters), the corresponding level of theory (LT) and basis set. Wall clock times (in minutes) for FC ( $\left.\mathrm{T}_{\mathrm{FC}}\right)$ and MTA ( $\mathrm{T}_{\mathrm{MTA}}$ ) vibrational IR and Raman spectral computations. See text for details.

\begin{tabular}{|c|c|c|c|c|}
\hline & System & LT/Basis & $\mathbf{T}_{\mathbf{F C}}$ & $\mathbf{T}_{\text {MTA }}$ \\
\hline \multirow[t]{7}{*}{ Vibrational IR } & $\left(\mathrm{CO}_{2}\right)_{25}$ & HF/aug-cc-pVDZ & $2851^{\mathrm{a}}$ & $5700^{c}$ \\
\hline & $\alpha-C y c l o d e x t r i n$ & B3LYP/6-31++G** & $6565^{\mathrm{a}}$ & $3191^{\mathrm{c}}$ \\
\hline & Subst-acetamide & B3LYP/6-311++G** & $2082^{\mathrm{b}}$ & $413^{\mathrm{b}}, 1024^{\mathrm{c}}$ \\
\hline & Vancomycin & B3LYP/6-31++G** & - & $19506^{\mathrm{b}}$ \\
\hline & Fc@CB[7] & $\omega \mathrm{B} 97 \mathrm{X} / 6-31+\mathrm{G}^{*}$ & - & $17954^{b}$ \\
\hline & $\mathrm{Bz}-\left(\mathrm{H}_{2} h\right)_{8}$ & MP2/aug-cc-pVDZ & $1738^{\mathrm{a}}$ & $947^{\mathrm{b}}$ \\
\hline & $\beta$-Carotene & MP2/aug-cc-pVDZ & - & $34960^{\mathrm{a}}$ \\
\hline \multirow[t]{2}{*}{ Vibrational Raman } & $\alpha-C y c l o d e x t r i n$ & B3LYP/6-31++G** & - & $2480^{c}$ \\
\hline & Subst-acetamide & B3LYP/6-311++G** & $1903^{b}$ & $1392^{\mathrm{c}}$ \\
\hline
\end{tabular}

Wall clock time on: 16 core machine, ${ }^{\mathrm{a}} 8$ core machine ${ }^{\mathrm{b}}$ and 4 core machine ${ }^{\mathrm{c}}$ respectively. 
quantum chemical investigations of even medium-sized molecular systems (typically containing $\sim 100$ atoms) at high-level theories such as DFT/MP2 and/or those employing large basis set, are prohibitively difficult employing any multi-core/high performing nodes. Hence for embarking upon such theoretical investigations, the inexpensive fragmentation-based alternative of using off-the-shelf multi-core machines offers a great advantage.

MTA offers a solution to this formidable challenge of $a b$ initio level IR/Raman spectra calculation of large molecules. In the present study, we have described a home-built, fragmentation-based MTA software meant for this purpose. For some of the test cases, we have compared the energetics and vibrational spectra generated by MTA with the respective FC counterparts. The energies for all the test cases (for which FC is feasible) are seen generally to match well their FC counterparts. Since the errors in MTA energies are indeed very small (sub millihartree), it is assured that the relative energies of isomers/conformers are well reproduced within MTA, obviating the need of Full calculations. ${ }^{13}$ Time advantage for the estimation of vibrational IR/Raman at HF/DFT level is comparatively smaller due to the inherent $\mathrm{O}\left(N^{3}\right.$ to $\left.N^{4}\right)$ scaling. However, in the case of a correlated method, e.g. MP2, significant time advantage is noticed. Otherwise, such FC is not feasible even on large contemporary hardware.

It is noteworthy that within the MTA procedure, computations on fragments are independent of each other, leading to an excellent parallel performance on a large number of nodes. This feature of MTA has been gainfully employed previously for MP2 level calculations on large water clusters. ${ }^{13}$ With a larger number of computational nodes, the performance of MTA to enable vibrational IR/Raman spectra of large systems is also expected to grow substantially. It is envisaged that with MTA running on a dozen multi-core computers, the calculations of vibrational IR/Raman spectra for spatially extended molecular systems containing $\sim 500$ atoms would be readily possible in the years to come. The present MTA software would be of interest to the molecular science community. It is planned to make the software available to them on request. Moreover, technical assistance would also be provided for the uninitiated users.

\section{Supplementary Information (SI)}

Sample input file, optimized Cartesian coordinates of all the test cases reported in the article at respective levels of theory, Graphical view of all the test molecules/cluster along with the superimposed MTA and FC spectra of the test systems. Supplementary Information is available at www.ias. ac.in/chemsci.

\section{Acknowledgements}

Support from the Center for Development of Advanced Computing (C-DAC), Pune is gratefully acknowledged. We also thank the DST-PURSE program for providing the Departmental computational facility.

\section{References}

1. Hochlaf M 2017 Advances in spectroscopy and dynamics of small and medium sized molecules and clusters Phys. Chem. Chem. Phys. 1921236

2. Kim H and Cho M 2013 Infrared probes for studying the structures and dynamics of biomolecules Chem. Rev. 113 5817

3. (a) Vass E, Hollósi M, Besson F and Buchet R 2003 Vibrational Spectroscopic detection of beta and Gammaturns in synthetic and natural peptides and proteins Chem. Rev. 103 1917; (b) Thomas Jr. G J 2002 New structural insights from Raman spectroscopy of proteins and their assemblies Biopolymers 67 214; (c) Lyon L A, Keating C D, Fox A P, Baker B E, He L, Nicewarner S R, Mulvaney S P and Natan M J 1998 Raman spectroscopy Anal. Chem. 70 341; (d) Fan Y, Ho J and Bettens R P A 2006 Approximating Coupled Cluster level vibrational frequencies with composite methods $J$. Phys. Chem. A 1102796

4. Friesner R A 2005 Ab initio quantum chemistry: Methodology and applications Proc. Natl. Acad. Sci. (U. S. A.) 1026648

5. Strout D L and Scuseria G E 1993 Quantitative study of the scaling properties of the Hartree-Fock method $J$. Chem. Phys. 1028448

6. Almlöf J, Faegri K and Korsell K 1982 Principles for a direct SCF approaches to LICAO-MO ab initio calculations J. Comp. Chem. 3385

7. Yoo S, Apra E, Zeng X C and Xantheas S S 2010 High level $a b$ initio electronic structure calculations of water clusters $\left(\mathrm{H}_{2} \mathrm{O}\right)_{16}$ and $\left(\mathrm{H}_{2} \mathrm{O}\right)_{17}$ : A new global minimum for $\left(\mathrm{H}_{2} \mathrm{O}\right)_{16}$ J. Phys. Chem. Lett. 13122

8. Werner H-J, Manby F R and Knowles P J 2003 Fast linear scaling second-order Møller-Plesset perturbation theory (MP2) using local and density fitting approximations $J$. Chem. Phys. 1188149

9. Weymuth T, Haag M P, Kiewisch K, Luber S, Schenk S, Jacob C R, Herrmann C, Neugebauer J and Reiher M 2012 MOVIPAC: Vibrational Spectroscopy with a robust meta-program for massively parallel standard and inverse calculations J. Compt. Chem. 332186

10. Bozkaya U and Sherrill C D 2017 Analytic energy gradients for the coupled-cluster singles and Doubles with Perturbative Triples method with the Density-Fitting Approximation J. Chem. Phys. 147044104

11. Guo Y, Becker U and Neese F 2018 Comparison and combination of direct and fragment based local correlation methods: Cluster in molecules and domain based local pair natural orbital perturbation and coupled cluster theories J. Chem. Phys. 148124117 
12. Sahu N and Gadre S R 2014 Molecular Tailoring Approach: A route for ab initio treatment of large clusters Acc. Chem. Res. 472739

13. Sahu N, Khire S S and Gadre S R 2015 Structures, energetics and vibrational spectra of $\left(\mathrm{H}_{2} \mathrm{O}\right)_{32}$ clusters: a journey from model potentials to correlated theory $\mathrm{Mol}$. Phys. 1132970

14. Collins M A and Bettens R P A 2015 Energy based molecular fragmentation methods Chem. Rev. 1155607

15. Raghavachari K and Saha A 2015 Accurate composite and fragment-based quantum chemical models for large molecules Chem. Rev. 1155643

16. Chung L W, Sameera W M C, Ramozzi R, Page A J, Hatanaka M, Petrova G P, Harris T V, Li X, Ke Z, Liu F, Li H-B, Ding L and Morokuma K 2015 The ONIOM method and its applications Chem. Rev. 1155678

17. Curtiss L A, Raghavachari K, Trucks G W and Pople J A 1991 Gaussian-2 theory for molecular energies of first- and second-row compounds J. Chem. Phys. 94 7221

18. Furtado J P, Rahalkar A P, Shanker S, Bandyopadhyay P and Gadre S R 2012 Facilitating minimum search for large water clusters at the MP2 level via. Molecular tailoring J. Phys. Chem. Lett. 32253

19. Ganesh V 2009 Meta Studio: A cross platform, programmable IDE for computational chemist J. Comput. Chem. 30661

20. Khire S S, Bartolotti L J and Gadre S R 2018 Harmonizing accuracy and efficiency: A pragmatic approach to fragmentation of large molecules J. Chem. Phys. 149 064112

21. Gaussian 16 Revision A.03 2016 Frisch M J, Trucks G W, Schlegel H B, Scuseria G E, Robb M A, Cheeseman J R, Scalmani G, Barone V, Petersson G A, Nakatsuji H, Li X, Caricato M, Marenich A V, Bloino J, Janesko B G, Gomperts R, Mennucci B, Hratchian H P, Ortiz J V, Izmaylov A F, Sonnenberg J L, Williams-Young D, Ding F, Lipparini F, Egidi F, Goings J, Peng B, Petrone A, Henderson T, Ranasinghe D, Zakrzewski V G, Gao J, Rega N, Zheng G, Liang W, Hada M, Ehara M, Toyota K,
Fukuda R, Hasegawa J, Ishida M, Nakajima T, Honda Y, Kitao O, Nakai H, Vreven T, Throssell K, Montgomery J A, Peralta Jr J E, Ogliaro F, Bearpark M J, Heyd, J J, Brothers E N, Kudin K N, Staroverov V N, Keith T A, Kobayashi R, Normand J, Raghavachari K, Rendell A P, Burant J C, Iyengar S S, Tomasi J, Cossi M, Millam J M, Klene M, Adamo C, Cammi R, Ochterski J W, Martin R L, Morokuma K, Farkas O, Foresman J B and Fox D J Gaussian, Inc., Wallingford CT.

22. Deshmukh M M, Bartolotti L J and Gadre S R 2011 Intramolecular hydrogen bond energy and cooperative interactions in $\alpha-, \beta$-, and $\gamma$-cyclodextrin conformers $J$. Comput. Chem. 322996

23. El-Azab A S, Mary Y S, Panicker C Y, Abdel-Aziz A A M, El-Sherbeny M A and Alsenoy C V 2016 DFT and experimental (FT-IR and FT-Raman) investigation of vibrational spectroscopy and molecular docking studies of 2-(4-oxo-3-phenethyl-3,4-dihydroquinazolin2-ylthio)-N-(3,4,5-trimethoxyphenyl) acetamide J. Mol. Struct. 1113133

24. Bykov D, Petreko T, Izsak R, Kossmaan S, Becker U, Valeev E and Neese F 2015 Efficient implementation of the analytic second derivatives of Hartree-Fock and hybrid DFT energies: a detailed analysis of different approximations Mol. Phys. 1131961

25. Nagy P R, Samu G and Kallay M 2016 An integral-direct linear-scaling second-order Møller-Plesset approach $J$. Chem. Theory Comput. 124897

26. Takeuchi H 2008 Geometry optimization of carbon dioxide clusters $\left(\mathrm{CO}_{2}\right)_{\mathrm{n}}$ for $4 \leq \mathrm{n} \leq 40$ J. Phys. Chem. A 112 7492

27. Miliordos E, Apra E and Xantheas S S 2016 A new, dispersion-driven intermolecular arrangement for the benzene-water octamer complex: Isomers and analysis of their vibrational spectra J. Chem. Theory Comput. 12 4004

28. Rao S S, Lande D N and Gejji S P 2016 Density functional theory investigations on binding and spectral features of complexes of ferrocenyl derivatives with cucurbit[7]uril J. Mol. Liq. 216298 